\title{
China-Japan Port Networks Suitable for Short Sea
}

\section{Shipping}

\author{
Jun Tong and Yutaka Watanabe \\ Graduate School, Tokyo University of Marine Science and Technology, Tokyo 135-8533, Japan
}

\begin{abstract}
During the last few decades, China and Japan have become mutually dependent economically. Most significantly, trade volume by container transportation between two countries has increased considerably. However, in situations of short distance, container transportation entails the important shortcomings of costly investment for land and infrastructure at ports as well as time-consuming complex cargo-handling operations. The distances of China and Japan routes are much shorter than those transoceanic sea routes used for container transportation, e.g., between Europe and Asia. In Europe, short sea shipping by RORO (roll-on/roll-off) or ferries is well networked among countries because of their proximity. Therefore, it is difficult for container transportation to avoid the effects of cost and inefficiencies described above. Accordingly, short sea shipping should be introduced properly between China and Japan because the distances separating the countries are similar to those of existing European short sea shipping networks. This paper presents an exploration of the possibility of introducing better short sea shipping networks between China and Japan. First, data related to short sea shipping in Europe were surveyed. The data include short sea shipping operators and regions with local ports, but also include regional populations, regional GDPs (Gross Domestic Product), number of regional tourists, and total regional freight tonnage. From analyzing the characteristics of short sea shipping networks, two major groups of the short sea shipping networks were found: one-to-one port networks and one-to-more port networks. Finally, geographical conditions of port locations between China and Japan were examined to ascertain whether any future port combinations can be suitable for expanding short sea shipping networks. Results show that port combinations among Okinawa in Japan, Taiwan, and southeastern China conformed to the one-to-more port networks. A short sea shipping network already exists between Okinawa and Taiwan. This paper reports the means of future expansion of the network to China.
\end{abstract}

Key words: Port networks, short sea shipping, regional transportation, distance range, tourism, cargo handling, container, logistics.

\section{Introduction}

Along with development of global production networks, marine transportation has also tended to become more globalized, as described by Notteboom [1]. Container transportation remains the main mode of marine transportation of goods. However, along with construction of port networks, short sea shipping is increasingly used for shorter distance between countries.

For short sea shipping, the main ship tonnage is $1,000 \sim 10,000$, with drafts ranging from around $2.5 \mathrm{~m}$ to $8.8 \mathrm{~m}$. Principally, transfers of wet and dry bulk cargoes (grain, tertilizers, steel, coal, salt, stone, scrap

Corresponding author: Yutaka Watanabe, Dr., professor, research fields: port logistics, intermodal transportation and safety engineering. and minerals, etc.) are done, in addition to those of containers and passengers [2].

Short sea shipping in Europe accounts for approximately $40 \%$ of all freight moved there. Cargo chiefly passes through the central region of Europe on rivers but not on oceans. Over the past several decades, the term of short sea shipping has broadened to include point-to-point cargo movements on inland waterways and inland to ocean ports for transhipment over oceans [3].

At the same time, short sea shipping has developed to some degree in the US. From the perspective of alleviating congestion, decreasing air pollution, and overall cost savings to the shipper and a government, short sea shipping is far more efficient and cost-effective than road transport. Moreover, it is 
much less prone to theft and damage.

China and Japan have become mutually dependent economically as the trade volume by container transportation between the countries has increased prodigiously. Nevertheless, container transportation networking for shorter distances has not occurred because of the necessary costly investments for land and infrastructure in ports and time-consuming complex cargo-handling operations. The distances separating China from Japan are short compared to those of transoceanic sea routes used for container transportation among the US, Europe, and Asia. Consequently, Ducruet [4] presented the view that potential network port cities can be evaluated by consideration of micro-scale (local environments) and macro-scale (regional patterns) factors. The present study chooses analyses of the port networks from macro-scale perspective and introduces better short sea shipping networks between China and Japan.

\section{Assumption and Methodology}

\subsection{Assumption of Heterogeneous Port Networks for Short Sea Shipping}

Global port networks linking economically developed regions that are mutually distant are generally connected by container shipping because of substantial cargo demand by trade between the regions. However, some economically developing regions might exist in proximity to those economically developed regions. Local port networks by RORO (roll-on/roll-off) or ferry shipping might be well adapted to such regional transportation because of the shorter distances with less or unstable cargo demand, as shown in the upper part of Fig. 1. Each port network is homogeneous depending on the shipping patterns, e.g., container, RORO, or ferry. If a shipping line calls for all the ports in the upper part of Fig. 1, then three port networks are needed, separately employing several ships on each network. That prospect presents risk to the shipping line.
However, geographic or economic niche markets of port networks might be found by which a shipping line can benefit from cargo demand for both economically developed regions and economically developing regions on a certain port network. When developing a geographic region that is located efficiently between two economically developed regions in terms of time or cost, the shipping line might be able to combine a global port network with different local port networks on the certain port network, as shown in the lower part of Fig. 1. This network of heterogeneous ports has different homogeneous port networks. Therefore, it is far superior for the shipping line than the case shown in the upper part of Fig. 1.

\subsection{Methodology for Evaluating Future Port Networks for Short Sea Shipping between China and Japan}

Based on the assumption presented above, this paper presents an examination of a methodology proposed by the following processes as depicted in Fig. 2:

(1) Assume advantages of heterogeneous port networks compared to homogeneous port networks;

(2) Analyze present port networks of short sea shipping in Europe because it is the world's largest

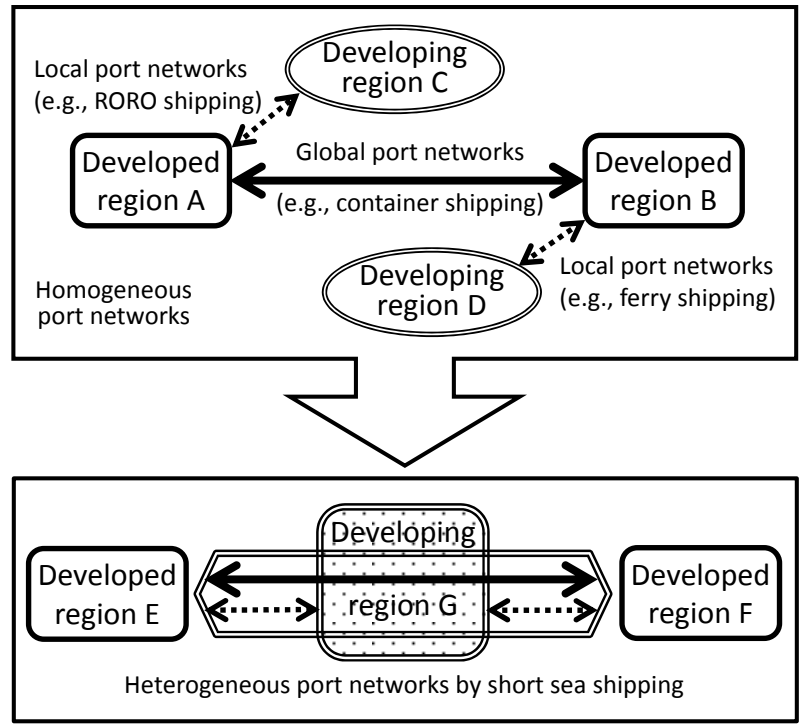

Fig. 1 Niche market of heterogeneous port networks for short sea shipping. 

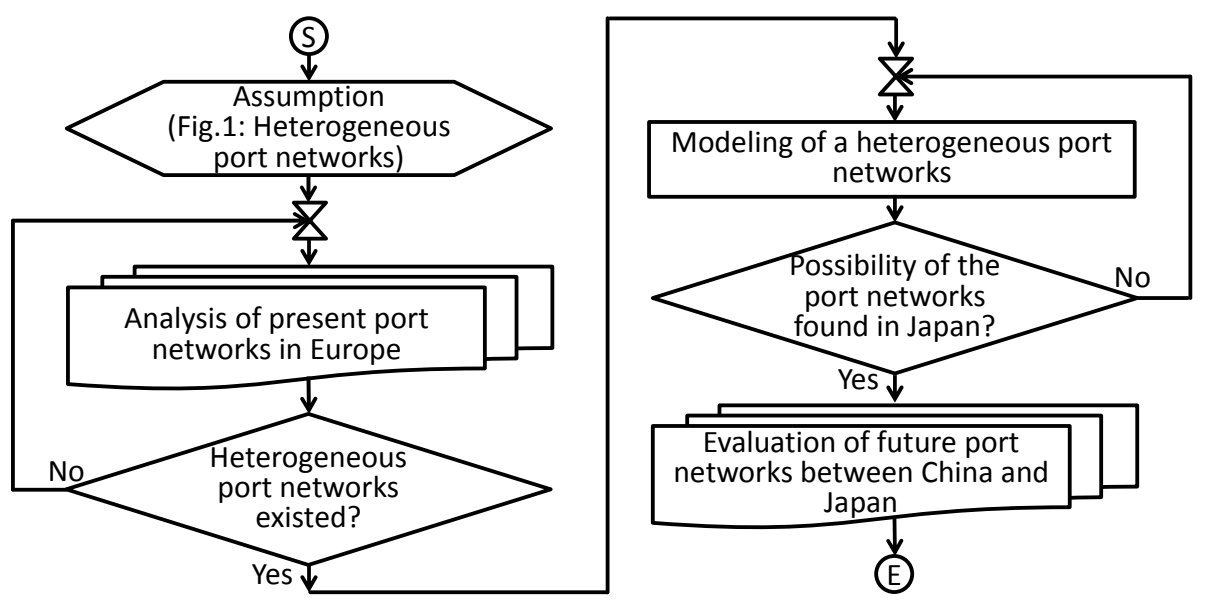

Fig. 2 Methodology for evaluating short sea shipping port networks suitable for expansion between China and Japan.

short sea shipping markets;

(3) Whether or not a heterogeneous port networks exist in Europe as assumed by (1);

(4) Model a heterogeneous port network that is applicable for short sea shipping between China and Japan;

(5) Find suitable port networks of short sea shipping between China and Japan, as modeled by (4);

(6) Evaluate possible future port networks of short sea shipping between China and Japan.

\section{Data Collection of European Short Sea Shipping}

\subsection{Well-Developed Networks of Short Sea Shipping in Europe}

Most European countries are well networked for short sea shipping because of their close mutual proximity. The salient benefits of short sea shipping in Europe portrayed in the figure are explainable by comparison with container transportation for transoceanic shipping routes. Ships used for short sea shipping are typically RORO or ferries [5]. Therefore, ports need less infrastructure for cargo handling equipment such as cranes. It is also an important benefit of RORO and ferries that cargoes on board are mobile, such as cars, trucks, and trailers. Therefore, the turnaround times of both the ships and the cargoes in ports are shorter than those for container transportation, for which containers must be stacked at container terminals in ports for days to weeks to match the timing of delivery or picking up of the containers by their shippers. Moreover, less space is needed in ports for short sea shipping because the wheeled cargoes can leave the ports soon after being discharged from the ships. In contrast, container terminals need more space in ports to accommodate a substantial number of containers, thereby meeting the timing needs of the shippers [6].

For China and Japan, Table 1 presents some short sea shipping routes in the Mediterranean by distance for illustration. Fig. 3 shows short sea shipping networks among countries according to data in Table 1. Short sea shipping is better at producing a network among neighboring regions at close distances when local ports are available for each. However, ports are not well equipped with infrastructure such as container terminals. The distances of China and Japan approximate those in the Mediterranean, as portrayed in Fig. 3. Therefore, suitable characteristics of short sea shipping in Europe can be found for possible new port networks for short sea shipping between China and Japan.

\subsection{Major Short Sea Shipping Operators in Europe}

Table 2 presents major operators of short sea shipping in Europe including all the networks presented 
Table 1 Examples of short sea shipping routes in the Mediterranean (listed by distance).

\begin{tabular}{llllll}
\hline No. & Region & Port & Region & Port & Distance (km) \\
\hline 1 & Andalucia, Spain & Algeciras & Ciudad Autonoma de Ceuta (ES), Spain & Ceuta & 25.56 \\
2 & Andalucia, Spain & Algeciras & Tangier-Tetouan & Tangier Med & 35.74 \\
3 & Campania, Italy & Napoli & Campania, Italy & Casamicciola & 60.00 \\
4 & Corsica, France & Calvi & Provence-Alpes-Cote d'Azur, France & Nice & 140.75 \\
5 & Corsica, France & Bastia & Toscana, Italy & Livorno & 143.57 \\
6 & Corsica, France & Bastia & Toscana, Italy & Piombino & 154.18 \\
7 & Andalucia, Spain & Almeria & Oriental, Morocco & Nador & 203.35 \\
8 & Andalucia, Spain & Almeria & Tlemcen, Algeria & Ghazaouet & 238.35 \\
9 & Cataluna, Spain & Barcelona & IIIes Balears, Spain & Ibiza & 243.35 \\
10 & Corsica, France & Bastia & Provence-Alpes-Cote d'Azur, France & Nice & 250.02 \\
11 & Andalucia, Spain & Almeria & Ciudad Autonoma de Melilla (ES), Spain & Melilla & 251.87 \\
12 & Cataluna, Spain & Barcelona & IIIes Balears, Spain & Formentera & 295.02 \\
13 & Corsica, France & Bastia & Liguria, Italy & Savona & 295.39 \\
14 & Corsica, France & Ajaccio & Provence-Alpes-Cote d'Azur, France & Toulon & 309.14 \\
15 & Cataluna, Spain & Barcelona & Languedoc-Roussillon, France & Sete & 319.56 \\
16 & Corsica, France & Ajaccio & Provence-Alpes-Cote d'Azur, France & Nice & 335.68 \\
17 & Andalucia, Spain & Almeria & Oran, Algeria & Oran & 350.03 \\
18 & Toscana, Italy & Livorno & Sardegna, Italy & Golfo Aranci & 362.64 \\
19 & Corsica, France & Bastia & Provence-Alpes-Cote d'Azur, France & Toulon & 387.07
\end{tabular}

Source: RO-RO \& Ferry Atlas Europe 2014/15 [7].

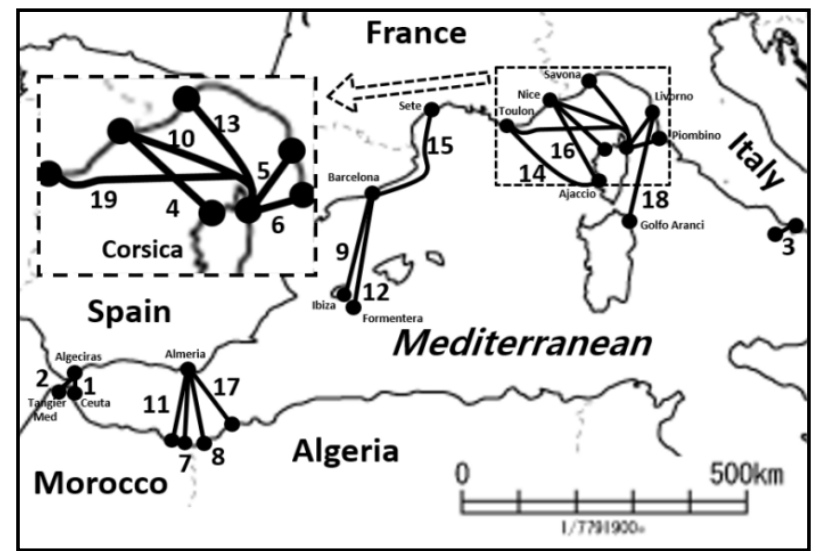

Fig. 3 Networks of short sea shipping route in Mediterranean.

Source: Table 1.

in Fig. 3. Numbers of regions and their local ports and combinations among the ports are sufficiently varied to show characteristics that are suitable for short sea shipping between China and Japan.

The prominent characteristics presented in Table 2 are that substantial capacity of passengers as well as cars and trucks are secured by the operators. Therefore, sufficient demand exists for short sea shipping in
Europe not only for pure logistics but also for passenger transportation, i.e., higher potential might exist for short sea shipping for tourism.

\subsection{Fundamental Activities in Regions Related to Short Sea Shipping}

In all, 56 regions have 86 local ports networked by the operators presented in Table 2, as shown in Table 3. Because it is natural to regard fundamental activities in regions as a driving force for short sea shipping, information related to the regional economy must be analyzed. For this study, population (inhabitants), GDP (Gross Domestic Product) and the number of tourists per inhabitant in each region together with total freight tonnage on routes of short sea shipping connected to each local port were collected for Europe in publicly available statistics. The dataset is presented in Table 3, in which the number of tourists per inhabitant was calculated by dividing the number of tourists by the population. 
Table 2 Major RORO and ferry operators in Europe.

\begin{tabular}{|c|c|c|c|c|c|c|}
\hline No. & Operators & No. vessels & $\begin{array}{l}\text { Total gross tonnage } \\
(2014 \sim 2015)\end{array}$ & $\begin{array}{l}\text { Total No. passengers } \\
(2014 \sim 2015)\end{array}$ & $\begin{array}{l}\text { Total No. cars } \\
(2014 \sim 2015)\end{array}$ & $\begin{array}{l}\text { Total No. trucks } \\
(2014 \sim 2015)\end{array}$ \\
\hline 1 & Stena Line & 24 & 749,998 & 23,801 & 6,470 & 151 \\
\hline 2 & P \& O Ferries & 16 & 496,495 & 16,448 & 7,288 & 484 \\
\hline 3 & DFDS Seaways & 11 & 296,394 & 12,726 & 3,935 & 230 \\
\hline 4 & Color Line & 6 & 246,770 & 10,004 & 2,644 & 0 \\
\hline 5 & Grandi Navi Veloci & 6 & 245,429 & 11,893 & 1,744 & 0 \\
\hline 6 & Brittany Ferries & 9 & 238,256 & 14,737 & 4,617 & 65 \\
\hline 7 & $\begin{array}{l}\text { Corsica Sardinia } \\
\text { Ferries }\end{array}$ & 11 & 222,837 & 18,435 & 5,770 & 0 \\
\hline 8 & Trasmediterranea & 14 & 214,7111 & 12,330 & 3,269 & 0 \\
\hline 9 & Irish Ferries & 5 & 149,250 & 6,300 & 5,385 & 0 \\
\hline 10 & SNAV & 6 & 134,776 & 7,482 & 2,392 & 236 \\
\hline 11 & Nel Lines & 10 & 70,179 & 10,601 & 2,519 & 0 \\
\hline 12 & LD Lines & 2 & 54,318 & 492 & 195 & 0 \\
\hline 13 & Condor Ferries & 4 & 11,264 & 2,523 & 625 & 0 \\
\hline
\end{tabular}

Source: Refs. [8-10].

Table 3 Fundamental activities in regions related to short sea shipping in Europe.

\begin{tabular}{|c|c|c|c|c|c|c|}
\hline No. & Region & Port & $\begin{array}{l}\text { Freight tonnage } \\
\text { (thousand tonnes) }\end{array}$ & $\begin{array}{l}\text { Population } \\
\text { (inhabitants) }\end{array}$ & GDP (Euro) & No. tourists \\
\hline 1 & Zuid-Holland, Netherlands & Rotterdam & 404,829 & $3,552,407$ & 32,000 & $2,079,393$ \\
\hline 2 & Zuid-Holland, Netherlands & Hoek of Holland & 404,829 & $3,552,407$ & 32,000 & $2,079,393$ \\
\hline 3 & Andalucia, Spain & Algeciras & 119,589 & $8,377,810$ & 18,300 & $9,584,247$ \\
\hline 4 & Andalucia, Spain & Almeria & 119,589 & $8,377,810$ & 18,300 & $9,584,247$ \\
\hline 5 & Andalucia, Spain & Cadiz & 119,589 & $8,377,810$ & 18,300 & $9,584,247$ \\
\hline 6 & Noord-Holland, Netherlands & Amsterdam & 93,453 & $2,709,822$ & 36,600 & $3,014,630$ \\
\hline 7 & $\begin{array}{l}\text { East Yorkshire and Northern } \\
\text { Lincolnshire, UK }\end{array}$ & Hull & 82,990 & 919,611 & 20,100 & $1,143,000$ \\
\hline 8 & $\begin{array}{l}\text { Provence-Alpes-Cote d'Azur, } \\
\text { France }\end{array}$ & Nice & 81,595 & $4,927,578$ & 26,000 & $11,004,030$ \\
\hline 9 & $\begin{array}{l}\text { Provence-Alpes-Cote d'Azur, } \\
\text { France }\end{array}$ & Toulon & 81,595 & $4,927,578$ & 26,000 & $11,004,030$ \\
\hline 10 & Sicilia, Italy & Palermo & 79,053 & $4,999,854$ & 16,300 & $2,548,463$ \\
\hline 11 & Haute-Normandie, France & Le Havre & 76,984 & $1,844,097$ & 24,000 & $1,938,264$ \\
\hline 12 & Haute-Normandie, France & Dieppe & 76,984 & $1,844,097$ & 24,000 & $1,938,264$ \\
\hline 13 & Liguria, Italy & Genoa & 69,359 & $1,567,339$ & 26,700 & $2,215,890$ \\
\hline 14 & Liguria, Italy & Savona & 69,359 & $1,567,339$ & 26,700 & $2,215,890$ \\
\hline 15 & Comunidad Velenciana, Spain & Valencia & 68,559 & $5,009,650$ & 21,200 & $5,536,438$ \\
\hline 16 & Cataluna, Spain & Barcelona & 67,908 & $7,514,991$ & 28,400 & $8,346,741$ \\
\hline 17 & Nord-Pas-de-Calais, France & Calais & 54,238 & $4,048,230$ & 22,100 & $3,087,397$ \\
\hline 18 & Nord-Pas-de-Calais, France & Dunkerque & 54,238 & $4,048,230$ & 22,100 & $3,087,397$ \\
\hline 19 & Sardegna, Italy & Golfo Aranci & 52,133 & $1,637,846$ & 19,300 & $1,247,003$ \\
\hline 20 & $\begin{array}{l}\text { West Wales and The Valleys, } \\
\text { UK }\end{array}$ & Fishguard & 49,420 & $1,936,283$ & 16,100 & $5,113,000$ \\
\hline 21 & $\begin{array}{l}\text { West Wales and The Valleys, } \\
\text { UK }\end{array}$ & Holyhead & 49,420 & $1,936,283$ & 16,100 & $5,113,000$ \\
\hline 22 & $\begin{array}{l}\text { West Wales and The Valleys, } \\
\text { UK }\end{array}$ & Pembroke & 49,420 & $1,936,283$ & 16,100 & $5,113,000$ \\
\hline 23 & Västsverige, Sweden & Gothenburg & 48,955 & $1,892,328$ & 29,600 & $4,030,458$ \\
\hline 24 & Västsverige, Sweden & Strömstad & 48,955 & $1,892,328$ & 29,600 & $4,030,458$ \\
\hline 25 & Västsverige, Sweden & Varberg & 48,955 & $1,892,328$ & 29,600 & $4,030,458$ \\
\hline
\end{tabular}


(Table 3 continued)

\begin{tabular}{|c|c|c|c|c|c|c|}
\hline No. & Region & Port & $\begin{array}{l}\text { Freight tonnage } \\
\text { (thousand tonnes) }\end{array}$ & $\begin{array}{l}\text { Population } \\
\text { (inhabitants) }\end{array}$ & GDP (Euro) & No. tourists \\
\hline 26 & Attiki, Greece & Piraeus & 47,332 & $3,961,122$ & 26,900 & $1,141,018$ \\
\hline 27 & Attiki, Greece & Lavrio & 47,332 & $3,961,122$ & 26,900 & $1,141,018$ \\
\hline 28 & $\begin{array}{l}\text { Hampshire and Isle of Wight, } \\
\text { UK }\end{array}$ & Portsmouth & 44,058 & $1,908,498$ & 25,600 & $2,798,000$ \\
\hline 29 & Lietuva, Lithuania & Klaipeda & 41,033 & $3,003,641$ & 16,900 & $1,090,318$ \\
\hline 30 & Sydsverige, Sweden & Karlskrona & 38,021 & $1,405,912$ & 26,800 & $2,169,464$ \\
\hline 31 & Sydsverige, Sweden & Trelleborg & 38,021 & $1,405,912$ & 26,800 & $2,169,464$ \\
\hline 32 & Sydsverige, Sweden & Karlshamn & 38.021 & $1,405,912$ & 26,800 & $2,169,464$ \\
\hline 33 & Pomorskie, Poland & Gdynia & 37,452 & $2,283,500$ & 15,600 & $1,511,595$ \\
\hline 34 & Kent, UK & Dover & 36,431 & $1,739,957$ & 21,200 & $1,486,000$ \\
\hline 35 & Schleswig-Holstein, Germany & Kiel & 34,765 & $2,837,641$ & 25,900 & $5,262,000$ \\
\hline 36 & Merseyside, UK & Liverpool & 32,924 & $1,508,892$ & 20,200 & $1,245,000$ \\
\hline 37 & $\begin{array}{l}\text { Prov. West-Vlaanderen, } \\
\text { Belgium }\end{array}$ & Zeebrugge & 31,974 & $1,173,818$ & 28,500 & $1,730,961$ \\
\hline 38 & Toscana, Italy & Livorno & 30,770 & $3,667,780$ & 27,600 & $5,489,961$ \\
\hline 39 & Toscana, Italy & Piombino & 30,770 & $3,667,780$ & 27,600 & $5,489,961$ \\
\hline 40 & Pay s de la Loire, France & Saint Nazaire & 29,219 & $3,630,780$ & 24,100 & $5,464,222$ \\
\hline 41 & Sy-ddanmark, Denmark & Esbjerg & 24,705 & $1,201,342$ & 27,800 & $1,151,795$ \\
\hline 42 & Sor-Ostlandet, Norway & Larvik & 24,604 & 949,963 & 27,500 & $2,658,344$ \\
\hline 43 & Sor-Ostlandet, Norway & Sandefjord & 24,604 & 949,963 & 27,500 & $2,658,344$ \\
\hline 44 & Northern Ireland, UK & Belfast & 23,226 & $1,818,935$ & 19,700 & $2,073,000$ \\
\hline 45 & Northern Ireland, UK & Larne & 23,226 & $1,818,935$ & 19,700 & $2,073,000$ \\
\hline 46 & $\begin{array}{l}\text { Mecklenburg-Vorpommern, } \\
\text { Germany }\end{array}$ & Sassnitz & 22,529 & $1,634,734$ & 21,100 & $6,501,273$ \\
\hline 47 & $\begin{array}{l}\text { Mecklenburg-Vorpommern, } \\
\text { Germany }\end{array}$ & Rostock & 22,529 & $1,634,734$ & 21,100 & $6,501,273$ \\
\hline 48 & Principado de Asturias, Spain & Gijon & 21,503 & $1,074,308$ & 22,700 & $1,501,806$ \\
\hline 49 & South Western Scotland, UK & Cairnryan & 19,845 & $2,332,454$ & 22,300 & $2,323,000$ \\
\hline 50 & South Western Scotland, UK & Troon & 19,845 & $2,332,454$ & 22,300 & $2,323,000$ \\
\hline 51 & Campania, Italy & Napoli & 18,258 & $5,764,424$ & 15,700 & $2,734,344$ \\
\hline 52 & Campania, Italy & Casamicciola & 18,258 & $5,764,424$ & 15,700 & $2,734,344$ \\
\hline 53 & Lazio, Italy & Civitavecchia & 17,765 & $5,500,022$ & 29,300 & $3,634,164$ \\
\hline 54 & Agder og Rogaland, Norway & Kristiansand & 17,606 & 728,934 & 36,500 & $1,925,614$ \\
\hline 55 & Jadranska Hrvatska, Croatia & Split & 14,839 & $1,410,551$ & 14,700 & $1,005,235$ \\
\hline 56 & Hovedstaden, Denmark & Copenhagen & 12,770 & $1,714,589$ & 38,300 & $1,196,177$ \\
\hline 57 & Midtjylland, Denmark & Grenaa & 12,682 & $1,266,682$ & 28,200 & 717,686 \\
\hline 58 & Marche, Italy & Ancona & 8,316 & $1,540,688$ & 25,500 & $1,854,613$ \\
\hline 59 & Nordjylland, Denmark & Frederikshavn & 7,917 & 579,996 & 27,500 & 574,229 \\
\hline 60 & Nordjylland, Denmark & Hirtshals & 7,917 & 579,996 & 27,500 & 574,229 \\
\hline 61 & $\begin{array}{l}\text { Northumberland and Tyne and } \\
\text { Wear, UK }\end{array}$ & Newcastle & 6,950 & $1,422,375$ & 20,800 & $2,032,000$ \\
\hline 62 & IIIes Balears, Spain & Ibiza & 6,871 & $1,100,715$ & 25,300 & $1,362,008$ \\
\hline 63 & IIIes Balears, Spain & Formentera & 6,871 & $1,100,715$ & 25,300 & $1,362,008$ \\
\hline 64 & Oslo og Akershus, Norway & Oslo & 5,641 & $1,169,539$ & 47,400 & $2,685,092$ \\
\hline 65 & Languedoc-Roussillon, France & Sete & 5,042 & $2,699,498$ & 20,900 & $6,540,839$ \\
\hline 66 & Bretagne, France & Roscoff & 4,939 & $3,239,659$ & 22,400 & $5,298,813$ \\
\hline 67 & Bretagne, France & St. Malo & 4,939 & $3,239,659$ & 22,400 & $5,298,813$ \\
\hline 68 & Cantabria, Spain & Santander & 4,803 & 592,383 & 23,500 & $1,268,303$ \\
\hline 69 & Lancashire, UK & Heysham & 4,035 & $1,463,495$ & 19,600 & $2,107,000$ \\
\hline
\end{tabular}


(Table 3 continued)

\begin{tabular}{|c|c|c|c|c|c|c|}
\hline No. & Region & Port & $\begin{array}{l}\text { Freight tonnage } \\
\text { (thousand tonnes) }\end{array}$ & $\begin{array}{l}\text { Population } \\
\text { (inhabitants) }\end{array}$ & GDP (Euro) & No. tourists \\
\hline 70 & Essex, UK & Harwich & 3,189 & $1,735,048$ & 21,500 & 961,000 \\
\hline 71 & Basse-Normandie, France & Cherbourg & 2,751 & $1,478,057$ & 21,200 & $2,928,513$ \\
\hline 72 & Basse-Normandie, France & Caen & 2,751 & $1,478,057$ & 21,200 & $2,928,513$ \\
\hline 73 & $\begin{array}{l}\text { Surrey, East and East Sussex, } \\
\text { UK }\end{array}$ & Newhaven & 2,696 & $2,755,118$ & 26,800 & $3,066,000$ \\
\hline 74 & Devon, UK & Plynouth & 2,374 & $1,139,062$ & 20,200 & $3,612,000$ \\
\hline 75 & $\begin{array}{l}\text { Ciudad Autonoma de Ceuta } \\
\text { (ES), Spain }\end{array}$ & Ceuta & 1,554 & 83,845 & 20,900 & 52,137 \\
\hline 76 & Corsica, France & Bastia & 1,554 & 318,316 & 22,800 & $1,746,324$ \\
\hline 77 & Corsica, France & Calvi & 1,554 & 318,316 & 22,800 & $1,746,324$ \\
\hline 78 & Corsica, France & Ajaccio & 1,544 & 318,316 & 22,800 & $1,746,324$ \\
\hline 79 & Notio Aigaio, Greece & Andros & 1,216 & 343,283 & 22,300 & 538,635 \\
\hline 80 & Notio Aigaio, Greece & Milos & 1,216 & 343,283 & 22,300 & 538,635 \\
\hline 81 & Notio Aigaio, Greece & Syros & 1,216 & 343,283 & 22,300 & 538,635 \\
\hline 82 & Notio Aigaio, Greece & Naxos & 1,216 & 343,283 & 22,300 & 538,635 \\
\hline 83 & $\begin{array}{l}\text { Anatoliki Makedonia, Thraki, } \\
\text { Greece }\end{array}$ & Kavala & 856 & 622,159 & 14,200 & 313,581 \\
\hline 84 & Dorset and Somerset, UK & Weymouth & 752 & $1,281,108$ & 21,100 & $3,188,000$ \\
\hline 85 & Dorset and Somerset, UK & Poole & 752 & $1,281,108$ & 21,100 & $3,188,000$ \\
\hline 86 & $\begin{array}{l}\text { Ciudad Autonoma de Melilla } \\
\text { (ES), Spain }\end{array}$ & Melilla & 604 & 81,792 & 19,000 & 36,718 \\
\hline
\end{tabular}

Source: Refs. [10-14].

Table 4 One-to-one port networks in Europe.

\begin{tabular}{|c|c|c|c|c|}
\hline No. & Region $\mathrm{A}$ & Port A & Region B & Port B \\
\hline 1 & $\begin{array}{l}\text { Northumberland and Tyne and } \\
\text { Wear, UK }\end{array}$ & Newcastle & Noord-Holland, Netherlands & Amsterdam \\
\hline 2 & $\begin{array}{l}\text { Surrey, East and East Sussex, } \\
\text { UK }\end{array}$ & Newhaven & Haute-Normandie, France & Dieppe \\
\hline \multirow{2}{*}{3} & \multirow{2}{*}{ Sydsverige, Sweden } & Karlskrona & Pomorskie, Poland & Gdynia \\
\hline & & Karlshamn & Lietuva, Lithuania & Klaipèda \\
\hline \multirow{2}{*}{4} & \multirow{2}{*}{ Vastsverige, Sweden } & Stromstad & Sor-Ostlandet, Norway & Sandefjord \\
\hline & & Varberg & Midtjylland, Denmark & Grenaa \\
\hline 5 & Campania, Italy & Napoli & Campania, Italy & Casamicciola \\
\hline 6 & Andalucia, Spain & Cadiz & Canary Islands & Arrecife \\
\hline 7 & Jadranska Hrvatska, Croatia & Split & Marche, Italy & Ancona \\
\hline
\end{tabular}

Source: Refs. [8-14].

\section{Analysis of Characteristics of European Short Sea Shipping}

\subsection{Combinations of Ports by Short Sea Shipping in} Europe

Analyses based on port of call services provided by each operator presented in Table 2 revealed 7 combinations of one-to-one port networks and 36 combinations of one-to-more ports networks. Table 4 shows the one-to-one port network. Table 5 shows the one-to-more port network. Numbers of ports were combined to produce one-to-more port networks or 2 5 destinations, as presented in Table 5. Hereinafter, a port connected with more than one port is designated as a master port. A port connected with such a master port is designated as a subordinate port, as expressed in the headings of Table 5. 
Table 5 One-to-more port networks in Europe.

\begin{tabular}{|c|c|c|c|c|c|c|c|c|c|}
\hline \multirow[b]{2}{*}{ No. } & \multicolumn{3}{|l|}{ Master } & \multicolumn{6}{|l|}{ Subordinates } \\
\hline & Region & Port & $\begin{array}{l}\text { No. } \\
\text { subordinate } \\
\text { ports }\end{array}$ & Region & Port & Region & Port & Region & Port \\
\hline \multirow{2}{*}{1} & \multirow{2}{*}{ Corsica, France } & \multirow{2}{*}{ Bastia } & \multirow[t]{2}{*}{ 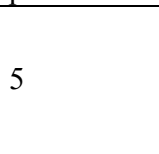 } & Toscana, Italy & Piombino & Toscana, Italy & Livorno & \multirow[t]{2}{*}{$\begin{array}{l}\text { Provence-Alpes-Cote } \\
\text { d'Azur, France }\end{array}$} & Nice \\
\hline & & & & Liguria, Italy & Savona & $\begin{array}{l}\text { Provence-Alpes-Cote d'Azur, } \\
\text { France }\end{array}$ & Toulon & & \\
\hline 2 & $\begin{array}{l}\text { Hampshire and Isle of } \\
\text { Wight, UK }\end{array}$ & Portsmouth & 4 & Cantabria, Spain & Santander & Haute-Normandie, France & Le Havre & $\begin{array}{l}\text { Basse-Normandie, } \\
\text { France }\end{array}$ & Caen \\
\hline \multirow[b]{2}{*}{3} & \multirow{2}{*}{$\begin{array}{l}\text { Basse-Normandie, } \\
\text { France }\end{array}$} & \multirow[b]{2}{*}{ Cherbourg } & \multirow[b]{2}{*}{4} & Dorset and Somerset, UK & Poole & \multirow[t]{2}{*}{ Southern and Eastern, Ireland } & & & \\
\hline & & & & $\begin{array}{l}\text { Southern and Eastern, } \\
\text { Ireland }\end{array}$ & Dublin & & & & \\
\hline \multirow{2}{*}{4} & \multirow{2}{*}{ Cataluna, Spain } & \multirow{2}{*}{ Barcelona } & \multirow{2}{*}{4} & Liguria, Italy & Genoa & \multirow[t]{2}{*}{ Languedoc-Roussillon, France } & \multirow[t]{2}{*}{ Sete } & \multirow[t]{2}{*}{ IIIes Balears, Spain } & \multirow[t]{2}{*}{ Ibiza } \\
\hline & & & & IIIes Balears, Spain & Formentera & & & & \\
\hline \multirow{2}{*}{5} & \multirow{2}{*}{ Andalucia, Spain } & \multirow{2}{*}{ Almeria } & \multirow{2}{*}{4} & Oriental & Nador & \multirow[t]{2}{*}{$\begin{array}{l}\text { Ciudad Autonoma de Melilla } \\
\text { (ES), Spain }\end{array}$} & Melilla & Tlemcen Province & Ghazaouet \\
\hline & & & & Oran Province & Oran & & & & \\
\hline 7 & $\begin{array}{l}\text { Dorset and Somerset, } \\
\text { UK }\end{array}$ & Poole & 3 & Basse-Normandie, France & Cherbourg & Bretagne, France & St Malo & $\begin{array}{l}\text { Principado de } \\
\text { Asturias, Spain }\end{array}$ & Gijon \\
\hline 8 & Southern and Eastern, & Dublin & 3 & Merseyside, UK & Liverpool & $\begin{array}{l}\text { West Wales and The Valleys, } \\
\text { UK }\end{array}$ & Holyhead & $\begin{array}{l}\text { Basse-Normandie, } \\
\text { France }\end{array}$ & Cherbourg \\
\hline 9 & Ireland & Rosslare & 3 & $\begin{array}{l}\text { West Wales and The } \\
\text { Valleys, UK }\end{array}$ & Fishguard & Basse-Normandie, France & Cherbourg & $\begin{array}{l}\text { West Wales and The } \\
\text { Valleys, UK }\end{array}$ & Pembroke \\
\hline 10 & $\begin{array}{l}\text { Provence-Alpes-Cote } \\
\text { d'Azur, France }\end{array}$ & Nice & 3 & Corsica, France & Calvi & Corsica, France & Ajaccio & Corsica, France & Bastia \\
\hline 11 & $\begin{array}{l}\text { Osloog Akershus, } \\
\text { Norway }\end{array}$ & Oslo & 3 & Nordjylland, Denmark & Frederikshavn & Schleswig-Holstein, Germany & Kiel & $\begin{array}{l}\text { Hovedstaden, } \\
\text { Denmark }\end{array}$ & Copenhagen \\
\hline 12 & Notio Aigaio, Greece & Syros & 3 & Notio Aigaio, Greece & Andros & Notio Aigaio, Greece & Milos & Notio Aigaio, Greece & Naxos \\
\hline 13 & Kent, UK & Dover & 2 & Nord-Pas-de-Calais, France & Calais & Nord-Pas-de-Calais, France & Dunkerque & & \\
\hline 14 & Devon, UK & Plymouth & 2 & Bretagne, France & Roscoff & Cantabria, Spain & Santander & & \\
\hline 15 & Essex, UK & Harwich & 2 & Zuid-Holland, Netherlands & $\begin{array}{l}\text { Hoek of } \\
\text { Holland }\end{array}$ & Syddanmark, Denmark & Esbjerg & & \\
\hline 16 & Merseyside, UK & Liverpool & 2 & $\begin{array}{l}\text { Southern and Eastern, } \\
\text { Ireland }\end{array}$ & Dublin & Northern Ireland, UK & Belfast & & \\
\hline
\end{tabular}


(Table 5 continued)

\begin{tabular}{|c|c|c|c|c|c|c|c|c|c|}
\hline \multirow[b]{2}{*}{ No. } & \multicolumn{3}{|c|}{ Master } & \multicolumn{6}{|c|}{ Subordinates } \\
\hline & Region & Port & $\begin{array}{l}\text { No. } \\
\text { subordinate } \\
\text { ports }\end{array}$ & Region & Port & Region & Port & Region & Port \\
\hline 17 & $\begin{array}{l}\text { East Yorkshire and } \\
\text { Northern } \\
\text { Lincolnshire, UK }\end{array}$ & Hull & 2 & $\begin{array}{l}\text { Prov. West-Vlaanderen, } \\
\text { Belgium }\end{array}$ & Zeebrugge & Zuid-Holland, Netherlands & Rotterdam & & \\
\hline 18 & Northern Ireland, UK & Larne & 2 & South Western Scotland, UK & Troon & South Western Scotland, UK & Cairnryan & & \\
\hline 19 & $\begin{array}{l}\text { South Western } \\
\text { Scotland, UK }\end{array}$ & Cairnryan & 2 & Northern Ireland, UK & Larne & Northern Ireland, UK & Belfast & & \\
\hline 21 & Bretagne, France & St Malo & 2 & Dorset and Somerset, UK & Poole & Dorset and Somerset, UK & Weymouth & & \\
\hline 22 & Corsica, France & Ajaccio & 2 & $\begin{array}{l}\text { Prov. West-Vlaanderen, } \\
\text { Belgium }\end{array}$ & Nice & $\begin{array}{l}\text { Provence-Alpes-Cote d'Azur, } \\
\text { France }\end{array}$ & Toulon & & \\
\hline 23 & $\begin{array}{l}\text { Provence-Alpes-Cote } \\
\text { d'Azur, France }\end{array}$ & Toulon & 2 & Corsica, France & Ajaccio & Corsica, France & Bastia & & \\
\hline 24 & Sydsverige, Sweden & Trelleborg & 2 & $\begin{array}{l}\text { Mecklenburg-Vorpommern, } \\
\text { Germany }\end{array}$ & Sassnitz & $\begin{array}{l}\text { Mecklenburg-Vorpommern, } \\
\text { Germany }\end{array}$ & Rostock & & \\
\hline 25 & Vastsverige, Sweden & Gothenburg & 2 & Schleswig-Holstein, Germany & Kiel & Nordjylland, Denmark & Fredrikshavn & & \\
\hline 27 & $\begin{array}{l}\text { Schleswig-Holstein, } \\
\text { Germany }\end{array}$ & Kiel & 2 & Vastsverige, Sweden & Gothenburg & Osloog Akershus, Norway & Oslo & & \\
\hline 28 & Liguria, Italy & Genoa & 2 & Cataluna, Spain & Barcelona & Sicilia, Italy & Palermo & & \\
\hline 29 & Sicilia, Italy & Palermo & 2 & Liguria, Italy & Genoa & Lazio, Italy & Civitavecchia & & \\
\hline 30 & Toscana, Italy & Livorno & 2 & Corsica, France & Bastia & Sardegna, Italy & Golfo Aranci & & \\
\hline 31 & & Piraeus & 2 & Voreio Aigaio, Greece & Vathy & Voreio Aigaio, Greece & Mytilene & & \\
\hline 32 & Attiki, Greece & Lavrio & 2 & $\begin{array}{l}\text { Anatoliki Makedonia, Thraki, } \\
\text { Greece }\end{array}$ & Kavala & Voreio Aigaio, Greece & Mesta & & \\
\hline 33 & Cantabria, Spain & Santander & 2 & $\begin{array}{l}\text { Hampshire and Isle of Wight, } \\
\text { UK }\end{array}$ & Portsmouth & Devon, UK & Plymouth & & \\
\hline 34 & Andalucia, Spain & Algeciras & 2 & $\begin{array}{l}\text { Ciudad Autonoma deCeuta } \\
\text { (ES), Spain }\end{array}$ & Ceuta & Tangier-Tetouan & Tangier Med & & \\
\hline 35 & IIIes Balears, Spain & Ibiza & 2 & Comunidad Valenciana, Spain & Valencia & Cataluna, Spain & Barcelona & & \\
\hline 36 & $\begin{array}{l}\text { Principado de } \\
\text { Asturias, Spain }\end{array}$ & Gijon & 2 & Dorset and Somerset, UK & Poole & Pays de la Loire, France & Saint Nazaire & & \\
\hline
\end{tabular}

Source: Refs. [8-10]. 


\subsection{Correlation of Freight Tonnage with Regional} Economy

Short sea shipping is supported by economical activities in the short term. In the longer term, port networks of the short sea shipping might, however, be affected by political relations, historical background, military power, and other factors. Analyses therefore demand the use of a macro-scale approach by which variables are widely applicable to different economic areas and a micro-scale approach by which variables are specialized to regionally oriented matters. These analyses examine the former set of variables. Therefore, it is better to include universal variables that are applicable to both Europe and Asia. The authors selected population, GDP, and number of tourists as such universal variables, although other candidate variables can be considered.

Table 6 presents correlation coefficients of the freight tonnage of both the one-to-one and the one-to-more port networks with the population, GDP, and the number of tourists per inhabitant in the regions connected with the port networks, with the calculated $R^{2}$. The population was significant, but GDP was not significant for any port network. Each had few tourists per inhabitant. None was significant for one-to-more port networks.

Comparison of $R^{2}$ of the population as shown in Fig. 4 reveals that coefficients of one-to-more port networks were significant, as were those of one-to-one port networks. This result might be explained by Table 4: most networks appearing in the table have greater importance for connecting two countries as a corridor rather than for meeting demand for the regional economies of local ports. This reasoning matches the finding of a lack of significance of one-to-one port networks with both GDP per inhabitant and the number of tourists per inhabitant in regions connected by port networks. This rationale also matches the assumption shown in the lower part of Fig. 1 that one to more port networks might have heterogeneous networks driven by both the developed and the developing regions, which is caused the higher significance of the population than that of one to one port networks, as shown in Table 6 and Fig. 4. Because

Table 6 Correlation of short sea shipping with regional economy in Europe by $\boldsymbol{R}^{2}$.

\begin{tabular}{lll}
\hline & One-to-more port networks & One-to-one port networks \\
\hline Population (inhabitants) & 0.6277 & 0.4181 \\
GDP (per inhabitant) & 0.0383 & 0.0022 \\
No. tourists per inhabitant & 0.1064 & 0.0570 \\
\hline
\end{tabular}

Source: Refs. [8-14].

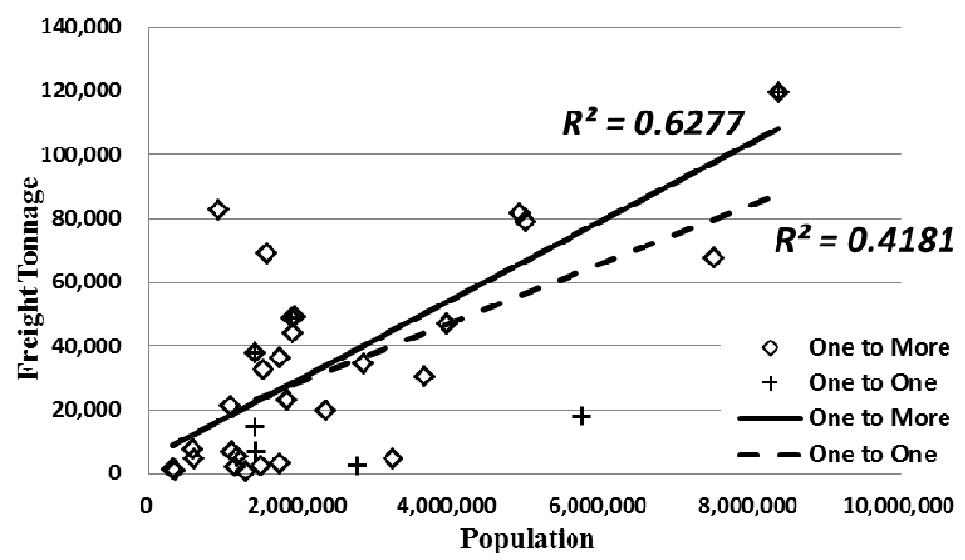

Fig. 4 Comparison of correlation $\left(R^{2}\right)$ with freight tonnage to population between one-to-one and one-to-more port networks.

Source: Refs. [8-14]. 
China and Japan are already connected by container transportation, maintaining such national economic corridors for decades, it is difficult for short sea shipping to be newly introduced between the countries under conditions of one-to-one port networks.

\subsection{Characteristics of Newly Introduced Short Sea Shipping between China and Japan}

Characteristics of short sea shipping in Europe suitable for China and Japan are found in the one-to-more port networks. However, too many networks exist, as shown in Table 5. It is therefore reasonable to infer that limited characteristics might be applicable for introducing new short sea shipping between China and Japan. When reviewed in Table 6, the population should be regarded as a general characteristic needed for all the one-to-more port networks because of the higher value of $R^{2}$. The number of tourists per inhabitant might affect a few networks, but apparently not all, because of the lower value of $R^{2}$. To verify this assumption, master ports and subordinate ports were compared with the number of tourists per inhabitant as shown in Fig. 5.

Two groups are clearly evident in the figure: one has a greater number of networks ranging widely in the figure; the other has only a few networks concentrated at the master ports but lower at the subordinate ports. These are the networks connected to Corsica of the Mediterranean as master ports. This is unexpected: an isolated region with low population such as Corsica has master ports. Therefore, master ports and subordinate ports were compared with the population, as shown in Fig. 6, which emphasizes the position of Corsica as highlighted by arrowed lines.

Two groups are shown in the figure as one distributed lower for master ports, but wider for subordinate ports and as other positions, mostly higher for the master ports. Corsica belongs to the former, and the lowest among master ports because Corsica is surrounded by populated and economically developed regions such as France and Italy, as shown at the right in Fig. 3.

Corsica is located intermediate between France and Italy. In addition to its position, the island has attractive tourism resources, although it is less populated. This is an ideal condition for RORO or ferry operators who wish to benefit from both the demand of pure logistics between the two countries and tourists to the island using a route connecting countries of destination at both sides calling for a master port at the island on the middle of the route, as illustrated at right in Fig. 3. By this configuration, the operators can maintain higher or stable booking orders for ships on the route throughout the year. This is the typical heterogeneous port network, as shown in the lower part of Fig. 1.

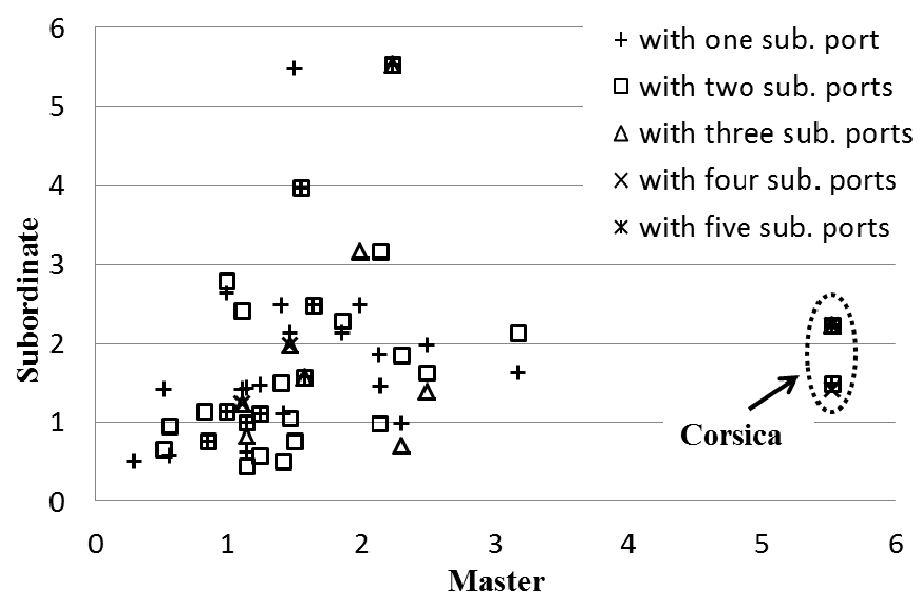

Fig. 5 Relation between master port and subordinate port on number of tourists per inhabitant. Source: Refs. [8-14]. 


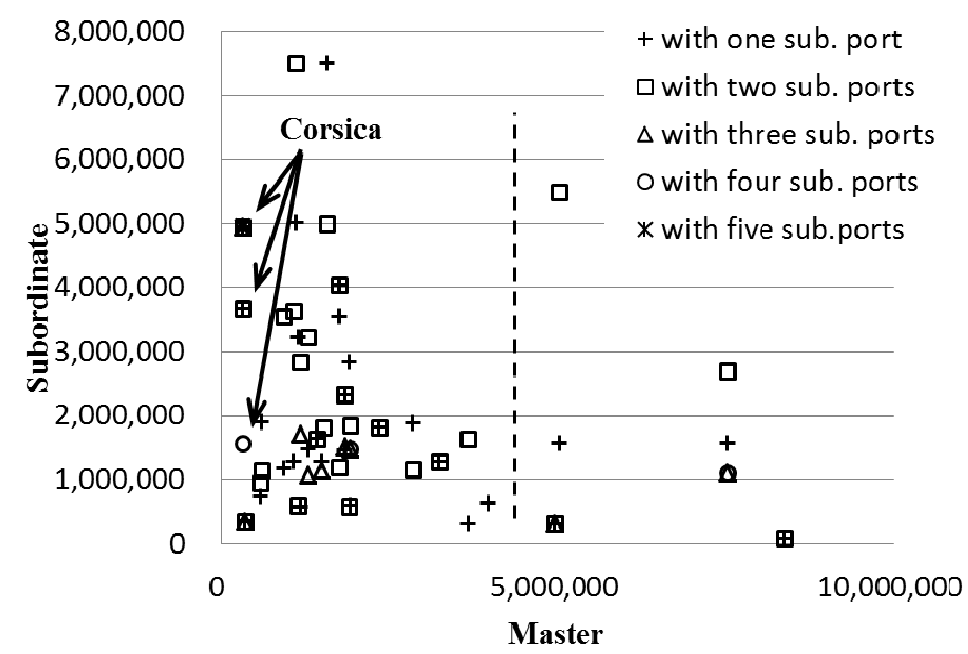

Fig. 6 Relation between master port and subordinate port on population.

Source: Refs. [8-14].

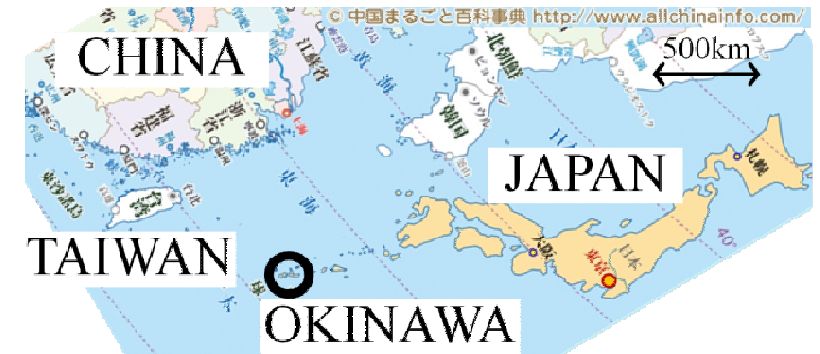

Fig. 7 Geographical proximities of Okinawa to China, Taiwan and Japan.

Source: All China Info [16].

\section{Possible One-to-More Port Networks of Short Sea Shipping between China and Japan}

\subsection{Similarity between Okinawa in Japan and Corsica in the Mediterranean}

Okinawa in Japan, located near the southwestern border of the country, can satisfy similar conditions to those of Corsica as presented below:

- Isolated island in the ocean;

- Surrounded by economically active countries nearby: China, Taiwan, and Japan;

- Valuable tourism resources exist on the island, which has various marine flora and fauna because of coral reefs near the island.

Fig. 7 presents the geographical proximities of Okinawa to the three countries.

\subsection{Verification on Benefits of Okinawa for Master Ports}

As the master port, Corsica is located intermediate between France and Italy (Fig. 3) with seven subordinate ports as shown in Table 5. To find the relation between Corsica with the capitals of the nearby countries, a comparison of the number of tourists per inhabitant, population and GDP between Corsica, Paris, and Rome is shown in Fig. 8. Results show that Corsica has numerous tourists per inhabitant but less population and GDP than either Paris or Rome.

To verify the advantage of Okinawa to have master ports on the one-to-more port networks of short sea shipping, the numbers of tourists per inhabitant, population and GDP were compared between Okinawa, Tokyo, and Taipei, as shown in Fig. 9.

It is readily apparent that Okinawa has great potential for tourism despite its lower population and GDP. For Okinawa, it has the same tendency as that of Corsica in terms of the number of tourists per inhabitant, the population and GDP comparing with the capitals. Therefore, Okinawa might benefit both from pure logistics among the three countries and from tourism from the three countries to the island, as the heterogeneous port network as shown in the lower 

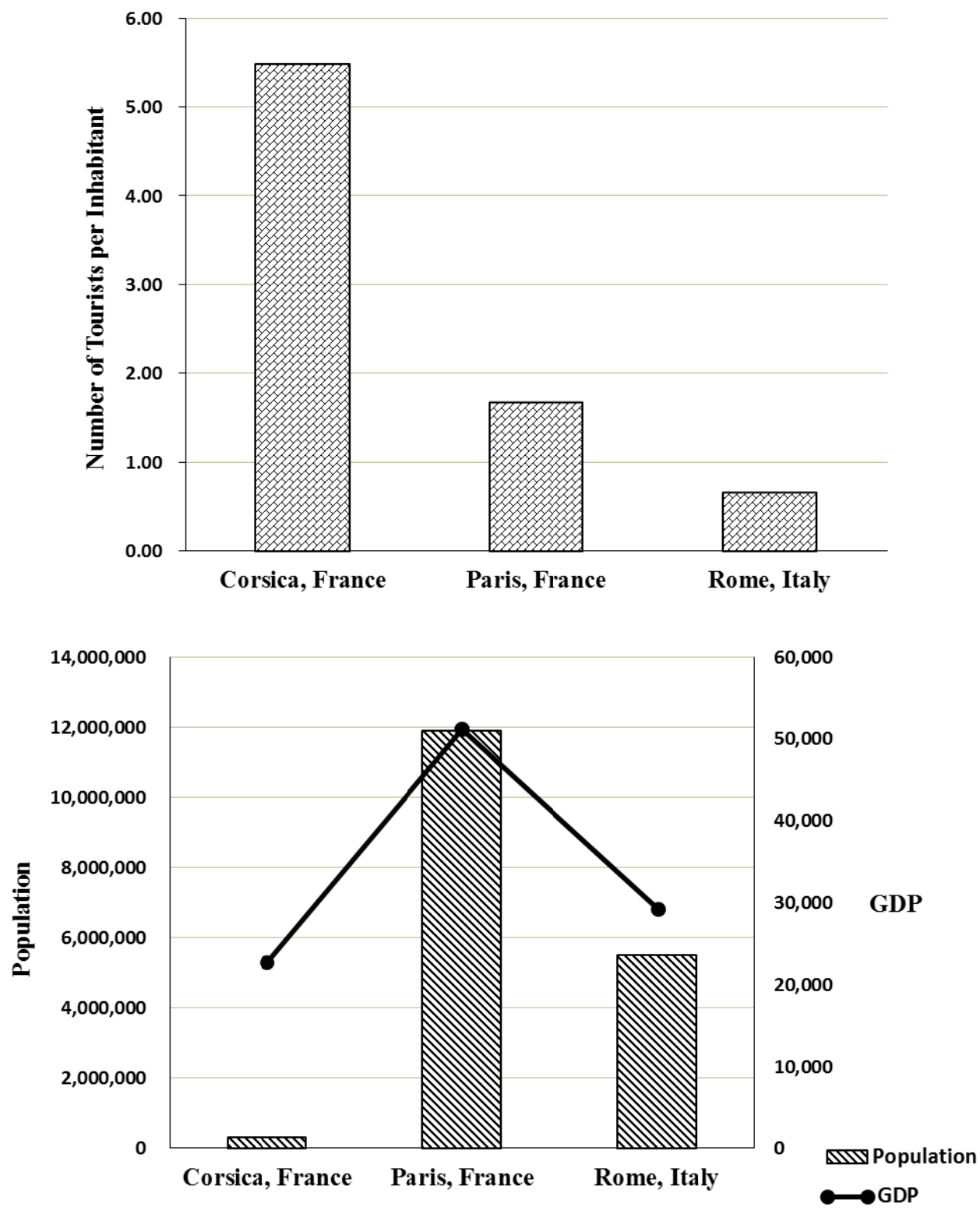

Fig. 8 Comparison of the number of tourists per inhabitant, population and GDP between Corsica, Paris, and Rome. Source: Refs. [11-14].

part of Fig. 1.

\subsection{Possible Expansion of Short Sea Shipping from} Okinawa to China

A domestic short sea shipping network has been operated by a Japanese operator for decades, connecting Okinawa with Tokyo and other economically developed regions throughout Japan. It was fortunate and good timing that the operator opened a new international short sea shipping route between Taiwan and Okinawa in June 2015: Ryukyu Kaiunkaisha [15]. This fact supports the results presented in the paper cited above. Furthermore, the new route of the operator is expected to be expanded southward to eastern coastal regions of China from Taiwan because of the results presented in this paper, as shown in Fig. 10. 

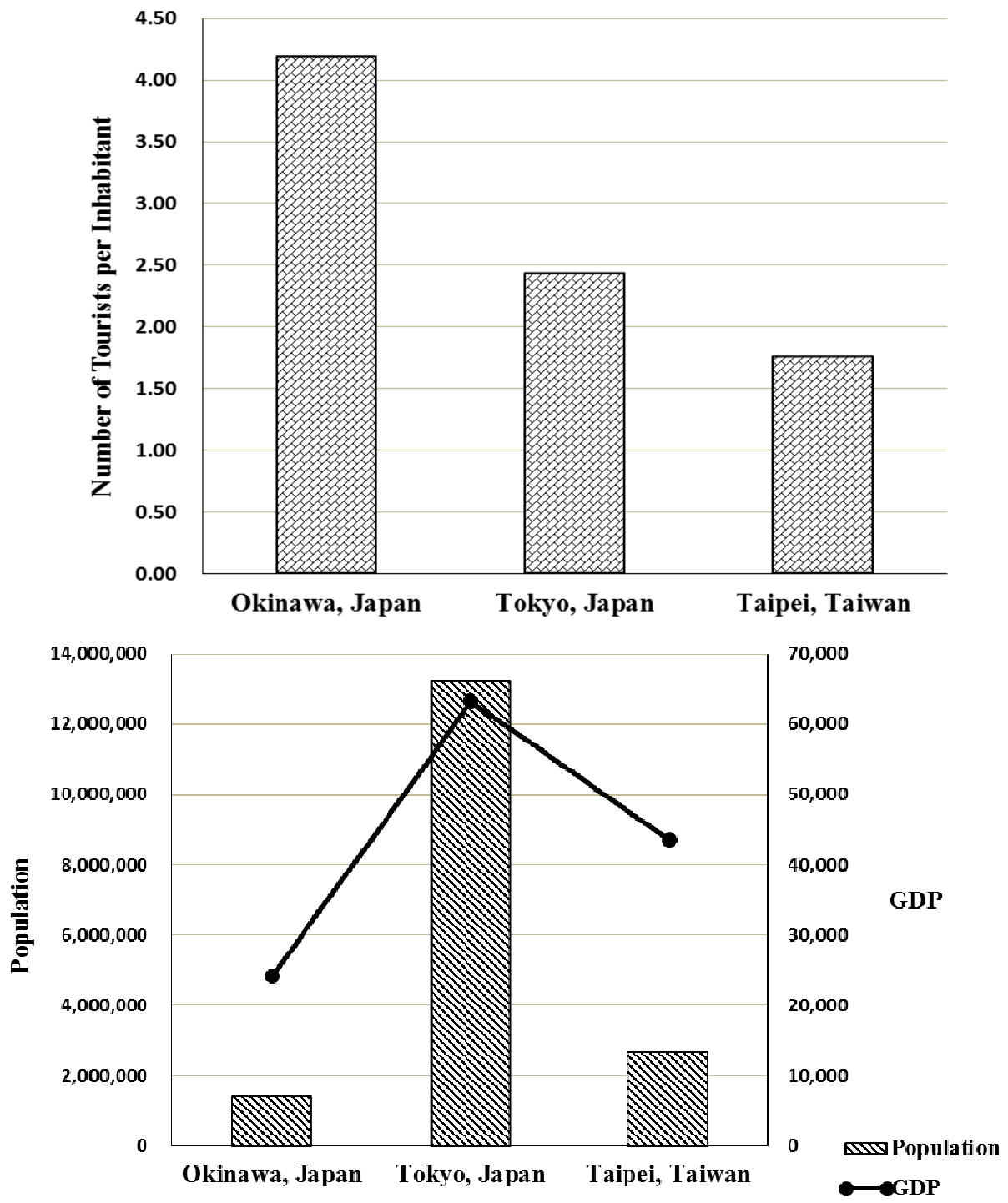

Fig. 9 Comparison of the number of tourists per inhabitant, population and GDP between Okinawa, Tokyo, and Taipei. Source: Refs. [17-25].

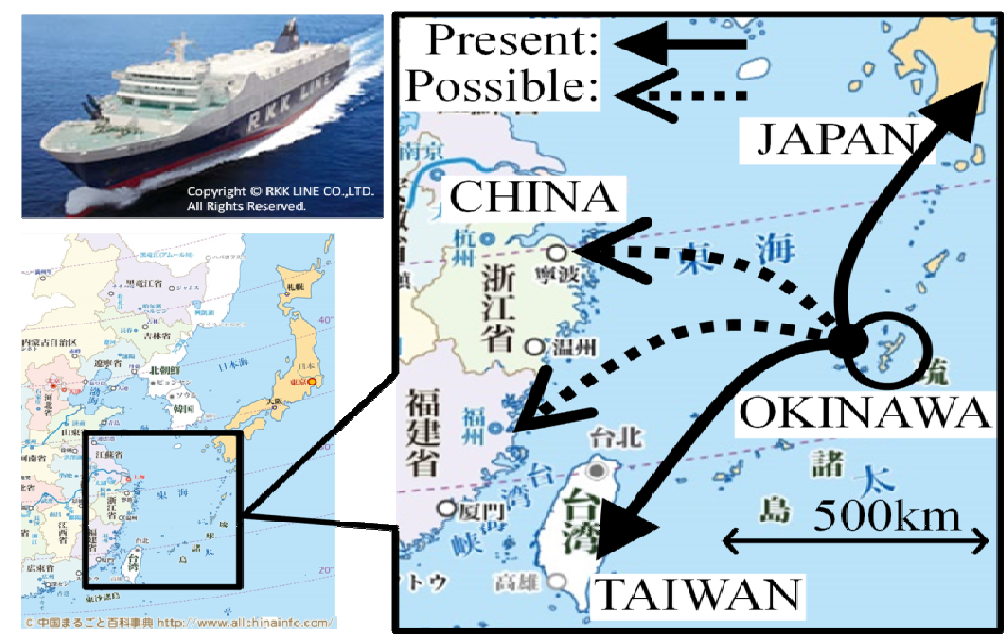

Fig. 10 Possible expansion of short sea shipping from Okinawa to China.

Source: Refs. $[15,16]$ 


\section{Conclusions}

Results of this study demonstrate that Okinawa can expand short sea shipping networks to China as a master port on the networks because of its similarity to Corsica in the Mediterranean. Corsica has benefited greatly from short sea shipping for many decades. Because of new short sea shipping routes opened between Taiwan and Okinawa this year, the conclusions presented herein are expected to contribute to mutual understanding between China and Japan of the importance and benefits of short sea shipping in the near future.

This paper mainly presents a macro-scale view to explain an exploration of the possibility of short sea shipping networks between China and Japan. For further research, the authors expect to examine microscale issues for comparison with macro-scale aspects to elucidate aspects such as regional matters, environmental factors, trade patterns, historical background.

\section{References}

[1] Theo, N. 2007. "Strategic Challenges to Container Ports in a Changing Market Environment." Transportation Economics 17: 29-52.

[2] European Shortsea Network. 2015. "About Shortsea, Definition, Sea-River Shipping in Europe." Accessed October 1, 2015. http://www.shortsea.info/more-infor mation-sea-river-shipping.html.

[3] European Shortsea Network. 2002. "About Shortsea, EU Commission, Leaflets, Shortsea Shipping." Accessed October 1, 2015. http://www.shortsea.info/medias/docu ments/leaflet_shortsea.pdf.

[4] César, D. 2006. "Port-City Relationships in Europe and Asia." Journal of International Logistics and Trade 4 (2): 13-35.

[5] Gardner, B., Lalwani, C., and Mangan, J. 2002. "Modelling Port/Ferry in RoRo Freight Transportation." International Journal of Transport Management 1: 15-28.

[6] Lago, A., Morales-Fusco, P., and Saurí, S. 2012. "Potential Freight Distribution Improvements Using Motorways of the Sea." Journal of Transport Geography 24: 1-11.

[7] Harbours Review. 2015. "RO-RO \& Ferry Atlas Europe 2014/15.” Accessed July 1, 2015. http://www.harboursreview.com/.

[8] Direct Ferries. 2014. "Ferry Companies." Accessed April 1, 2014. http://www.directferries.co.uk/operators.htm.

[9] Ferrylines.com. 2014. "Operators, Europe-International." Accessed July 1, 2014. http://www.ferrylines.com/en/ operators/europe-international/.

[10] Marine Traffic. 2014. "Vessels." Accessed June 1, 2014. http://www.marinetraffic.com/en/ais/index/ships/all.

[11] Eurostat. 2011. "Data, Database by Themes, Economy and Finance, National Accounts (Including GDP) (ESA95) (na), Annual National Accounts (nama), Regional Economic Accounts-ESA95 (nama_r_gdp), Gross Domestic Product(GDP) at Current Market Prices by NUTS 2 Regions (nama_r_e2gdp)." Accessed August 1, 2014. http://ec.europa.eu/eurostat/data/database.

[12] Eurostat. 2012. "Data, Database by Themes, Industry, Trade and Services, Tourism (Tour), Annual Data on Tourism Industries (tour_inda), Occupancy of Tourist Accommodation Establishments (tour_occ), Arrivals of Residents and Non-residents (tour_occ_a), Arrivals at tourist Accommodation Establishments by NUTS 2 regions (tour_occ_arn2)." Accesed June 1, 2015. http://ec.europa.eu/eurostat/data/database.

[13] Eurostat. 2012. "Data, Database by Themes, Population and Social Conditions, Population Change-Demographic Balance and Crude Rates at Regional Level (NUTS 3).” Accessed August 1, 2014. http://ec.europa.eu/eurostat/ data/database.

[14] Eurostat. 2012. "Data, Database by Themes, Transport, Maritime Transport, Maritime Transport-Regional Statistics (mar_rg), Maritime Transport of Freight by NUTS 2 Regions (tran_r_mago_nm).” Accessed August 1, 2014. http://ec.europa.eu/eurostat/data/database.

[15] RKK Line Co. Ltd. 2015. Homepage. Accessed July 1, 2015. http://www.rkkline.co.jp/index.html.

[16] All China Info. 2015. "Map of East Asia.” All China Info. Accessed July 1, 2015. http://www.allchinainfo.com/ map/asia-china/asia.

[17] Cabinet Office, Government of Japan. 2011. "Statistics, Data GDP." Accessed December 1, 2015. http://www.esri.cao.go.jp/jp/sna/data/data_list/kenmin/fil es/contents/main_h24.html.

[18] Okinawa Prefecture Government. 2011. "Okinawa Statistics, Economy Accounts, 2012.” Accessed December 1, 2015. http://www.pref.okinawa.jp/toukeika/ accounts/2012/acc4.pdf.

[19] Okinawa Prefecture Government. 2012. "Okinawa Statistics, Groups Search, Cutural Tourism Sports." Accessed December 1, 2015. http://www.pref.okinawa.lg. $\mathrm{jp} /$ site/bunka-sports/kankoseisaku/kikaku/statistics/tourist s/documents/h24nendo-gaikyou.pdf. 
[20] Okinawa Prefecture Government. 2012. "Okinawa Statistics, Population Estimates, 2012.” Accessed December 1, 2015. http://www.pref.okinawa.jp/toukeika/ estimates/2012/201212.pdf.

[21] Taipei Eonomy. 2015. "Article." Accessed October 1, 2015. http://www.taipeiecon.taipei/article_cont.aspx?Mm $\mathrm{mID}=1204 \& \mathrm{MSid}=655141564222712442 /$.

[22] The Japanese municipality of Okinawa Prefecture. 2013. "Population Estimates."Accessed July 1, 2015. http://www.pref.okinawa.jp/toukeika/estimates/2013/201 312.pdf.

[23] The Japanese municipality of Okinawa Prefecture. 2013.
"Tourists Estimates." Accessed July 1, 2015. http://www.pref.okinawa.jp/site/bunka-sports/kankoseisa $\mathrm{ku} / \mathrm{kikaku} /$ statistics/tourists/documents/h25gaikyou_1.pdf.

[24] Tokyo Convention \& Visitors Bureau. 2012. "Tourism Media, Tourist of Tokyo 2012." Accessed December 1, 2015. http://www.gotokyo.org/jp/administration/h25/doc uments/shiryou1.pdf.

[25] Tokyo Metropolitan Government. 2012. “About Our City, The Long-Term Vision for Tokyo. Tokyo's History, Geography, and Population, Population of Tokyo." Accessed December 1, 2015. http://www.metro.tokyo.jp/ ENGLISH/ABOUT/HISTORY/history03.htm. 